\title{
Study of Fault Diagnosis for Rolling Bearing Based on GA-BP Algorithm Yue Wen ${ }^{1, a}$, Minping Jia $^{2, b}$, Cheng Luo ${ }^{3, c}$ \\ ${ }^{1}$ School of Mechanical Engineering, Southeast University, Nanjing, 211189, China \\ ${ }^{2}$ School of Mechanical Engineering, Southeast University, Nanjing, 211189, China \\ ${ }^{3}$ School of Mechanical Engineering, Southeast University, Nanjing, 211189, China \\ awenyue2015@163.com, 'bmpjia@163.com, ${ }^{\mathrm{c}}$ cheng_chris@163.com
}

\section{Keywords: Genetic Algorithm; BP Neural Network; Fault Diagnosis; Rolling Bearing}

\begin{abstract}
With the development of modern technology, the diagnosis technology for mechanical equipment has attached more attentions. This paper mainly presents the fault diagnosing process for rolling bearing by using the hybrid GA-BP algorithm, which combines genetic algorithm (GA) with error back-propagation algorithm (BP). The weights and thresholds of BP neural network are optimized by using genetic algorithm firstly and then assigned to the BP neural network. With the using of proposed approach, the diagnosis performance of BP neural network can be improved. In this paper, the optimal nonlinear mapping relationship between fault types and fault symptoms of rolling bearings is obtained and then realize failure recognition of rolling bearings though the hybrid GA-BP algorithm. Finally, the results of the test experiment verifies the efficiency and accuracy of the proposed GA-BP algorithm.
\end{abstract}

\section{Introduction}

As an important economic loss and damage for the country, the failure of mechanical equipment, especially the damage of key mechanical component has been brought more attention. As a result, the mechanical equipment fault diagnosis technology is extremely important. Update to 2016, the failure of rotating machinery caused by rolling bearing faults accounted for $30 \%$. Therefore, the fault diagnosis for rolling bearings is essential. In recent years, artificial intelligence has been developed and artificial neural network is widely applied in many fields [1] including fault diagnosis as an original modeling technique. Artificial neural network is a mathematical model that imitates animals' neural network behavior characteristics to realize distributed parallel processing of information. Among several kinds of artificial neural network models, the application of BP neural network with a single hidden layer accounts for $90 \%$. However, the disadvantage of the conventional BP algorithm is that it is easy to fall into the local minimum and the convergence speed of the network weights and thresholds is too slow. GA algorithm has a strong ability of macroscopic search and can find the global optimal solution with large probability. The hybrid algorithm combines GA and BP algorithm provides a new approach for the fault diagnosis.

\section{Theories of BP Neural Network and Genetic Algorithm}

Artificial neural network (ANN) is a kind of information processing system, and the neuron is the basic component unit of neural network. Multiple neurons interconnected together, resulting in a neural network structure of adaptive nonlinear dynamic system. Artificial neural network is generally composed of input layers, hidden layers and output layers. In practice, the artificial neural network containing only one hidden layer is called BP neural network. A typical BP neural network model is shown in figure 1 below. 


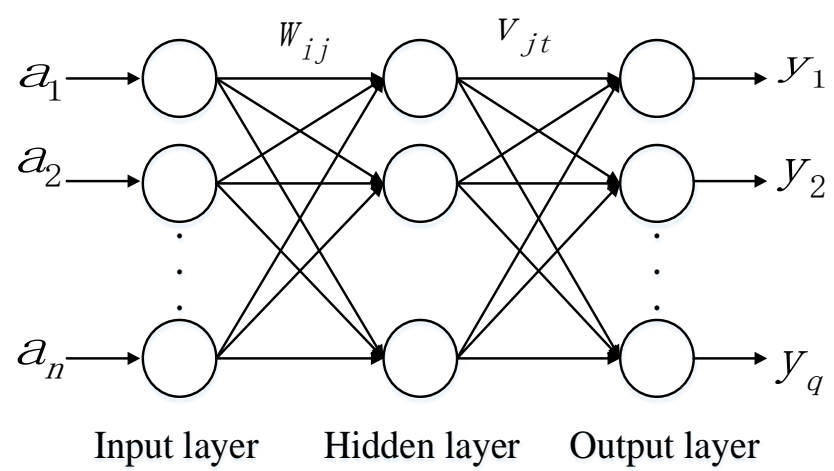

Fig. 1. Structure of BP neural network

BP neural network is a kind of supervised learning algorithm, which consists of two parts called forward propagation and feedback propagation [2]. In the process of forward propagation, the information of neurons for each layer only relates to the incoming information of neurons before this layer and influence the state of neurons followed this layer. When the actual output does not match the desired output, the work of this BP neural network will enter into the process of feedback propagation. In this process, the signal of error is returned through the original path, and the connection weights $w_{i j}$ and thresholds $v_{j t}$ of the network are adjusted simultaneously to get the optimal weights and thresholds, which resulting in the minimum error. Hence, the feedback propagation is viewed as the key step in the process of learning and training the BP neural network [3].

Genetic algorithm(GA) was first proposed by Professor John Holland of Michigan University in 1975, it is a random search method referenced to the law of biological evolution called "survival of the fittest" genetic mechanism and is widely used to solve the optimization problem. The genetic algorithm starts from any initial population and guides the search process to approach the region where the optimal solution is located through selection, crossover and mutation operations. The advantage of genetic algorithm is that the optimal solution or satisfactory solution can be obtained without traversing the whole search space, and the combinatorial explosion problem can be solved well [4]. The basic contents of the genetic algorithm include encoding, generating feasible initial population, determining the fitness function and determining the genetic operator. The figure 2 shows the flow chart of genetic algorithm.

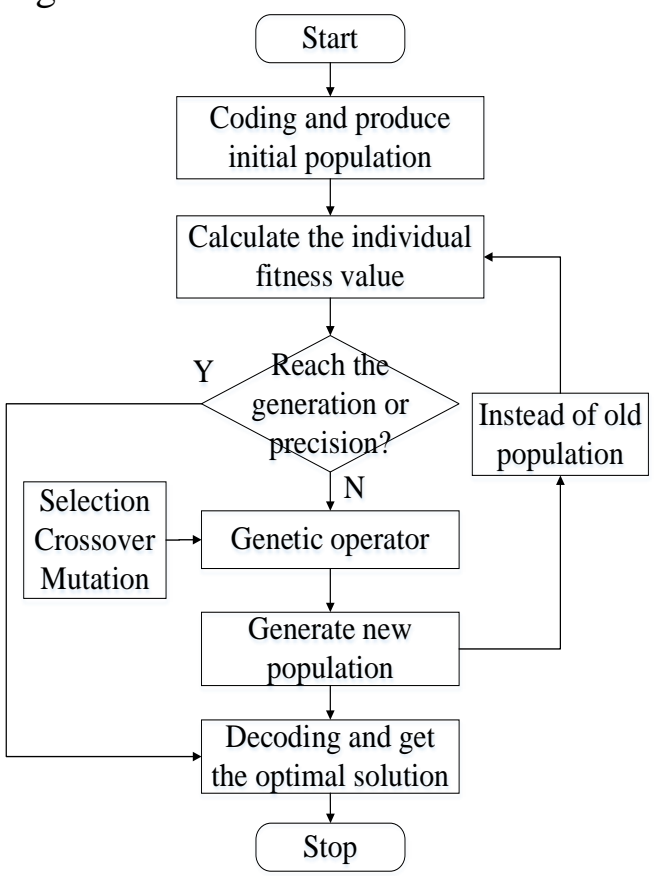

Fig. 2. Flow chart of genetic algorithm 


\section{The Rolling Bearing Fault Diagnosis Based on GA-BP Algorithm}

The weights and thresholds of the network are learned and trained by the conventional BP algorithm to get an optimal network structure, then the optimal nonlinear mapping relationship between the input and output data is obtained. However, the convergence speed of the weights and thresholds of BP algorithm is too slow and it is easy to fall into the local minimum. GA algorithm is a global optimization algorithm and the convergence speed is fast. Considering of the advantages of GA algorithm, GA-BP hybrid algorithm is proposed by combining GA algorithm and BP algorithm to provide a new approach for mechanical fault diagnosis [5][6]. GA-BP algorithm is used to optimize the weights and thresholds of traditional BP neural network though GA algorithm firstly and then assign the optimized values to the BP neural network.

When using BP neural network to recognize the pattern of mechanical failure types, the input neurons of neural network correspond to a set of characteristic vector, it reflects the fault symptoms of different fault types [7]. In this paper, wavelet analysis was used to obtain the characteristic vector. The output neurons of the neural network correspond to the fault types. The number of neurons in the input layer is equal to the number of elements of an input characteristic vector and the number of neurons in the output layer is defined as the number of the corresponding fault types for the training samples. In the process of fault diagnosis, in order to get the appropriate weights and thresholds to determine the neural network structure, several sets of fault samples are used to optimize the weights and thresholds by GA algorithm firstly. Then provides the optimized weights and thresholds for the BP network and train them again using BP algorithm to get the optimal nonlinear mapping relationship between fault types and fault symptoms. Finally, input a group of current fault feature vector into the BP neural network and output the corresponding fault types to realize failure recognition of mechanical equipment [8]. In this paper, the rolling bearing was seen as research object and through the fault diagnosis based on GA-BP algorithm, the rolling bearing fault types were recognized automatically. The flow chart of rolling bearing fault diagnosis based on GA-BP algorithm is shown in figure 3 below.

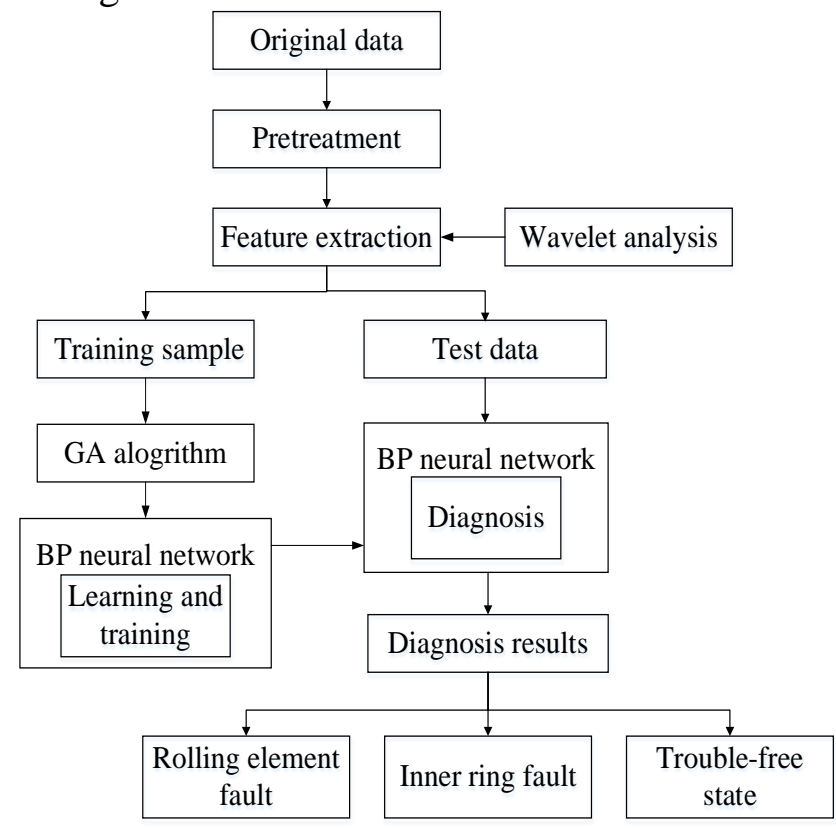

Fig. 3. The rolling bearing fault diagnosis flow chart based on GA-BP algorithm

In the validation test of the proposed GA-BP algorithm in this paper, the rolling bearing fault data from the bearing test center of Case Western Reserve University were used and the detail process is shown as follows:

(1)Zero mean process and normalize the raw data of rolling bearing;

(2)Analyze the data after pretreatment through wavelet analysis [9]. First, treat the data by wavelet decomposition and reconstruction, then calculate the energy of reconstructed signal to construct energy eigenvector and normalize it as the training samples and test data respectively; 
(3)Train the weights and thresholds by GA algorithm and assign the optimized values to the BP neural network;

(4)Train the weights and thresholds again through BP algorithm and determine the ultima network structure;

(5)Send the test data into the determined BP network to identify types of rolling bearing faults and realize the fault intelligent diagnosis [10].

\section{The Diagnosis Result and Analysis}

Based on the GA-BP algorithm for rolling bearing fault diagnosis in this paper, the data of rolling element fault, inner ring fault and trouble-free state were analyzed to verify the efficiency and accuracy of the proposed approach.

The target data corresponding to the input data of the training sample is a vector with 0 and 1 as elements. The number 1 represents failure occurred while 0 represents trouble-free state. The maximum training times of the neural network was set to 8000 and the minimum error was 0.01 . Tangent sigmoid function 'tansig' and linear function 'purelin' were selected as activation functions of the hidden layer and output layer, gradient descent function 'traingd' was selected as the training function of this neural network. The BP neural network model was built in this paper is shown in figure 4 below.

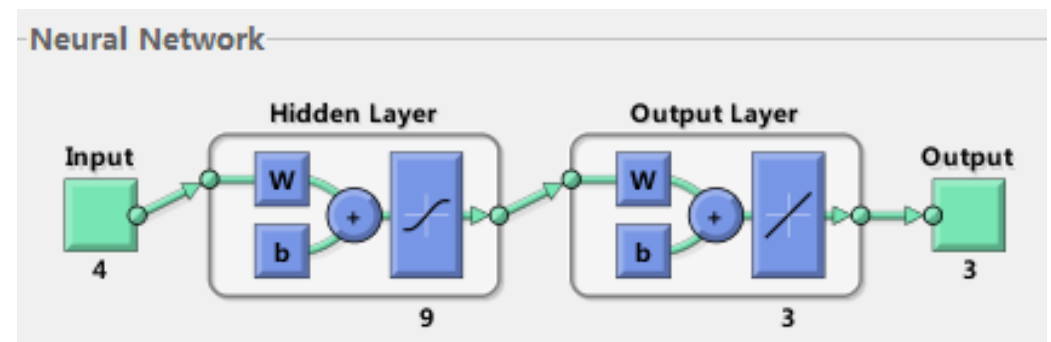

Fig. 4. The BP neural network structure

To prove the GA-BP algorithm is superior to conventional BP algorithm, a contrast experiment of BP algorithm and GA-BP algorithm was implemented.

Figure 5 showed the curve of mean square error based on BP algorithm. It showed that when training epochs up to the maximum 8000, the minimum mean square error (mse) value was 0.009 . The time of training process was $51 \mathrm{~s}$.

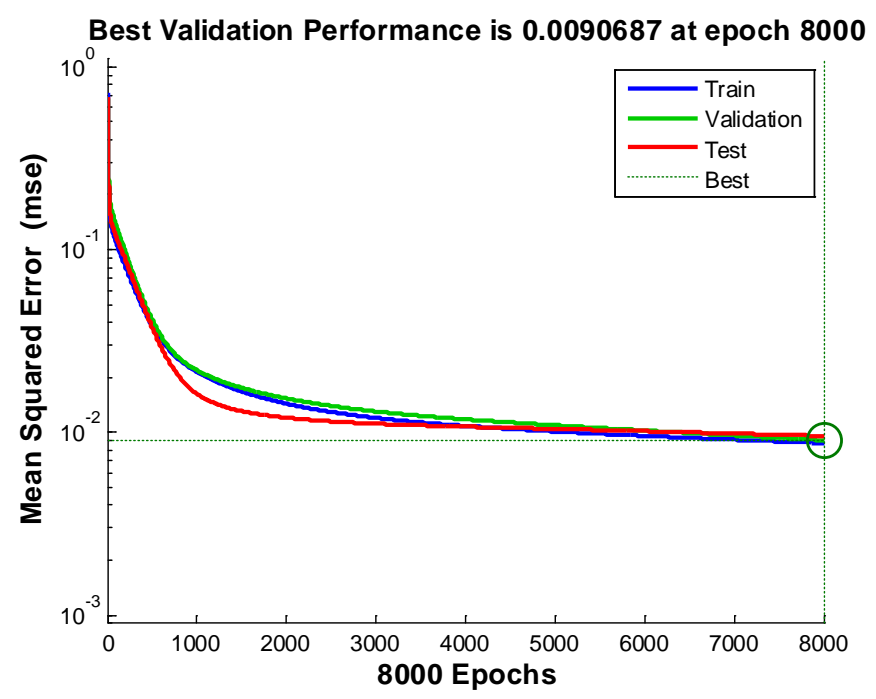

Fig. 5. The curve of mean square error based on BP algorithm

Figure 6 showed the curve of fitness function based on GA algorithm. The figure illustrated that the best fitness and mean fitness of each generation is stable after about 25 generations. 


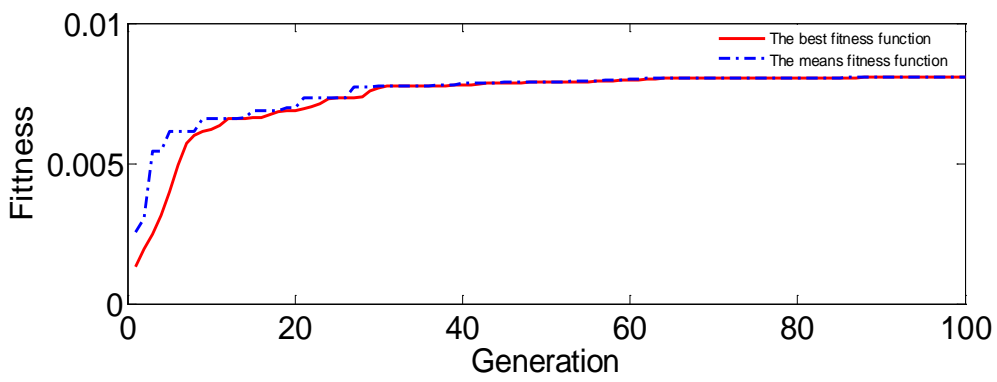

Fig.6. The curve of fitness function based on GA algorithm

Figure 7 showed the curve of mean square error based on GA-BP algorithm. It illustrated that when training epochs up to 3613, the minimum mean square error (mse) value was 0.010. The time of training process was 23s.

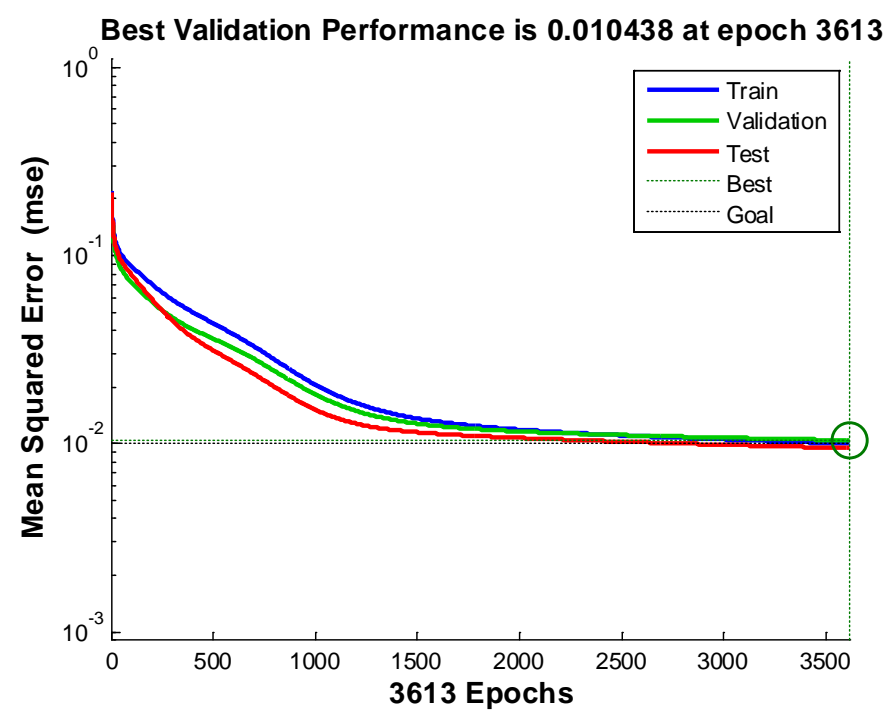

Fig. 7. The curve of mean square error based on GA-BP algorithm

At this point, the weights and thresholds of the BP network were adjusted to the optimal values through BP algorithm and GA-BP algorithm respectively. Input a set of test data of inner ring fault to the two determined BP network and the output results are shown in Table 1 below.

Table 1 Test Results Contrast

\begin{tabular}{cccc}
\hline Input data & Target output & $\begin{array}{c}\text { Output data based on } \\
\text { BP algorithm }\end{array}$ & $\begin{array}{c}\text { Output data based on } \\
\text { GA-BP algorithm }\end{array}$ \\
\hline 0.6569 & 0 & 0.0637 & -0.0771 \\
0.6971 & 1 & 0.9244 & 1.0605 \\
0.2835 & 0 & 0.0068 & 0.0042 \\
0.0460 & - & - & - \\
\hline
\end{tabular}

From the test results of BP algorithm and GA-BP algorithm, it shows that the output data based on the two methods approximated to theoretical output $[0,0,1]$, which represents inner ring fault occurred in the roller bearing. However, the processing time of GA-BP algorithm was 23s while the processing time of BP algorithm was 51s. So the convergence speed based on GA-BP algorithm is faster than BP algorithm. Therefore, the accuracy and efficiency of rolling bearing fault diagnosis based on GA-BP algorithm is verified.

\section{Conclusion}

In this paper, the theories of BP neural network and GA algorithm were studied firstly. Considering of the defects of conventional BP algorithm and the advantages of GA algorithm, the GA-BP algorithm combined these two algorithms is proposed to realize the failure recognition of 
roller bearings. GA algorithm is used to optimize the weights and thresholds of traditional BP neural network and then the optimized values are assigned to the BP neural network. Next, BP algorithm is used to train the weights and thresholds again to get the optimal nonlinear mapping relationship between failures and fault symptoms. The fault data from bearing test center of Case Western Reserve University were used in the verification test. Wavelet analysis method was used to extract the fault feature of the data to constructed the training samples and a set of test data. Comparing the results of BP algorithm and GA-BP algorithm, the efficiency and accuracy of GA-BP algorithm are verified and intelligent diagnosis of rolling bearing faults is realized.

\section{Acknowledgement}

The research described in this paper was financially supported by the National Natural Science Foundation of China (Project No. 51675098), Chinese Specialized Research Foundation for Doctoral program of Higher Education (Project No. 20130092110003) and Graduate student research innovation Foundation of Jiangsu province (Project No. SJLX16_0034).

\section{References}

[1] Wong,B.K.,T.A.Bodnovich and Y.Selvi. Neural network applications in business: A review and analysis of the literature (1988-1995). Decision Support Systems[J].1997. 19(4): p. 301-320.

[2] SUN, Y., et al. Improved BP Neural Network for Transformer Fault Diagnosis[J]. Journal of China University of Mining and Technology, 2007.17(1): p. 138-142.

[3] Kexin Yuan. Engine fault diagnosis research based on BP neural network[D]. Shandong, Shandong University, 2006.

[4] Ziegel E. Genetic Algorithms and Engineering Optimization[M]. Genetic algorithms and engineering design. Wiley, 2002:379-381.

[5] Zhen-Jiang L V, Tang J S. Research of Fan Fault Diagnosis Based on Genetic Algorithm[J]. Coal Engineering, 2015.

[6] Ping M A, Wang Y, Zhang J, et al. Using GA To Train The BP Neural Network Based MATLAB To Complete Fault Diagnosis[J]. Microcomputer Information, 2007, 23(28):142-144.

[7] Ling,L., Y.Huang and L.Qu. Research on Fault Diagnosis Based on BP Neural Network Optimized by Chaos Ant Colony Algorithm[J], in Advances in Swarm Intelligence,2010:p. 257-264.

[8] Subrahmanyam, M. and C. Sujatha. Using neural networks for the diagnosis of localized defects in ball bearings[J]. Tribology International, 1997. 30(10): p. 739-752.

[9] Bin, G.F., et al. Early fault diagnosis of rotating machinery based on wavelet packets-Empirical mode decomposition feature extraction and neural network[J]. Mechanical Systems and Signal Processing, 2012.27: p. 696-711.

[10] JiangFeng Luo. The application of wavelet packet and BP neural network in gearbox fault diagnosis[J]. Mechanical drive, 2007 (03) :84-86. 